\title{
Narcissus (Amaryllidaceae), la evolución de los polimorfismos florales y la conservación más allá de las "listas rojas"
}

\author{
Narcissus (Amaryllidaceae), the evolution of flower polymorphisms and conservation \\ beyond the "red lists"
}

JUAN ARROYO

Departamento de Biología Vegetal y Ecología, Universidad de Sevilla, Apartado 1095, 41080 Sevilla, España; e-mail: arroyo@us.es

\begin{abstract}
RESUMEN
Se realiza una revisión del significado del género Narcissus (Amaryllidaceae) para explicar los distintos modelos de evolución de la heterostilia, aportándose datos recientes que confirman el buen ajuste que muestra este género a los pasos propuestos en el modelo de Lloyd \& Webb (1992a, 1992b). En particular, y a falta de una filogenia que establezca fielmente las condiciones ancestrales y derivadas, existen especies de Narcissus que representan casi todos los estadios del modelo: monomorfismo floral con hercogamia de aproximación y compatibilidad genética, dimorfismo estilar, distilia y tristilia. El sistema de incompatibilidad es variable, pero parece que no es del tipo usual en las plantas tradicionalmente consideradas heterostilas (i.e., dialélico). Además de estos patrones macroevolutivos (ligados a especiación), se analizan variaciones a escala poblacional (microevolutiva) para explicar por qué el dimorfismo estilar es tan frecuente en este género y en particular por qué en casi todas las especies dimórficas predomina el morfo de estilo largo. Dado que Narcissus es un género con alta diversidad de especies, muchas de ellas endémicas, y aparece frecuentemente en las "listas rojas" de especies amenazadas y en peligro de extinción, se evalúan los criterios que se han seguido para incorporar estas especies. La mayor parte de las especies de Narcissus incluidas en las listas rojas pertenecen sólo a algunos grupos taxonómicos (secciones), que además son los menos diversos en cuanto a polimorfismos representados en los modelos de evolución de la heterostilia. Por otro lado, se revisa la información biológica contenida en algunas de las listas rojas, con objeto de poder utilizarla a su vez en la evaluación del modelo evolutivo citado. Con pocas excepciones, esta información no es útil por escasa o inexacta. Se sugiere que en los pasos siguientes a la elaboración de las listas rojas se tenga en cuenta la relevancia científica que las especies pueden tener como sistemas de estudio de procesos ecológicos y/o evolutivos de carácter más general. Idealmente, la información a obtener para las especies de las listas rojas debería constituir una Flora Biológica al modo en que viene haciéndose en las Islas Británicas o, más recientemente, en Europa central.
\end{abstract}

Palabras clave: cuenca mediterránea, diversidad, endemismo, geófito, heterostilia, sistemas de reproducción.

\begin{abstract}
In this review I address the significance of the genus Narcissus (Amaryllidaceae) as an ideal study case for the models of the evolution of heterostyly, supplying recent data which confirm a good fit to Lloyd \& Webb's (1992a, 1992b) model. In particular, there are species of Narcissus which represent ancestral and derived conditions suggested in that model, although a robust phylogeny is still needed to ascertain such a pattern. Hence, flower monomorphism with approach herkogamy and genetic compatibility, stylar dimorphism, distyly and tristyly are reported. The incompatibility system is variable, although the one typical of heterostylous plants (i.e., diallelic) is not found in Narcissus. In addition to these macroevolutionary patterns (associated to speciation), I analyse some variations at the population (i.e., microevolutionary) scale in order to explain why stylar dimorphism is so frequent in this genus, and particularly why the long-styled morph is more frequent in most species. Since Narcissus is a species-rich genus, including many endemic taxa, it has representatives in most «red lists» of threatened species within its distributional range. Therefore, I considered this genus appropriate for evaluating the criteria commonly used to incorporate species to these lists. Most red-listed Narcissus species belong to only a few taxonomic groups (sections), which, in turn, are the less diverse in flower polymorphisms meaningful to the models for the evolution of heterostyly. On the other hand, the biological information included in these lists is evaluated in order to its possible use in model testing. In most cases, this information is useless, since it is inexact or too vague. I suggest that, in further elaborations of red lists, the scientific relevance of species as study systems to understand general evolutionary and/or ecological processes should be considered. Ideally, the information to be obtained for these species should constitute a data base sufficient to elaborate a Biological Flora similar to the long since being established in the British Isles or the recently started one in central Europe.
\end{abstract}

Key words: breeding system, diversity, endemism, geophyte, heterostyly, Mediterranean basin. 


\section{INTRODUCCIÓN}

La biología de la conservación es una disciplina de carácter científico relativamente nuevo, aunque los antecedentes filosóficos sean mucho más antiguos. Es por naturaleza de carácter interdisciplinar al incluir ecología, sistemática y genética, entre otros campos. Por ello, los objetivos que pretende pueden ser múltiples y pertenecer a varias escalas. En uno de los textos más influyentes (Primack 1993) se definen dos objetivos básicos que encierran muchos otros: (i) la investigación del impacto humano en la diversidad biológica y (ii) el desarrollo de aplicaciones prácticas para prevenir la extinción de las especies. Estos planteamientos abren cuestiones adicionales, por ejemplo cómo definir y medir la diversidad biológica, si sólo la categoría de especie merece atención o si todas las especies la merecen por igual. Se han usado muchos argumentos para apoyar la conservación de las especies, pero interesa aquí resaltar uno de ellos: en palabras de Given (1994) “el valor científico de las plantas y la oportunidad de estudiar determinados procesos ecológicos (y evolutivos)". Obviamente, el proceso mejor ejemplificado es la extinción, pero no es el único.

La mayor parte de los estudios se dirigen al nivel de especie o de conjuntos de éstas (comunidades, floras, faunas, ecosistemas). Los niveles inferiores (subespecies, poblaciones, individuos, genes) raramente han sido considerados como objetivos en sí mismos (véase no obstante Solbrig 1991), aunque son muy tenidos en cuenta para explicar los procesos que conducen a la extinción. Desde un punto de vista práctico, esta aproximación se ve plasmada en la aparición de numerosas "listas rojas" de especies amenazadas. Estas listas en ocasiones incluyen poco más que los nombres de las especies y su distribución geográfica aproximada, pero más recientemente pretenden incluir información biológica detallada que pueda ser útil para el manejo y recuperación de tales especies (véase Given 1994 para ejemplos de diversos tipos de listas y para recomendaciones sobre la información biológica a incluir).

En cuanto a la diversidad biológica, ésta tiene un valor intrínseco que debe ser conservado (Primack 1993, Given 1994). Su definición y medición ha producido una abundante literatura, principalmente en el ámbito ecológico. En un contexto aplicado a conservación, diversidad biológica es casi siempre equivalente a diversidad de especies, aunque en ciertas ocasiones se entiende que esta última sólo captura parte de la información sobre diversidad: hay unas especies que aportan más a la diversidad que otras. Esta idea es antigua en el plano ecológico debido a la importancia diferente que presentan las especies en las redes tróficas en general y en las mutualistas en particular ("key stone species": Paine 1966, Gilbert 1980). Más recientemente y provocado por limitaciones prácticas en el número de especies y áreas a conservar, pero también por ciertas consideraciones teóricas (May 1990, Fiedler \& Ahouse 1991), se ha intentado evaluar la diversidad filogenética que incorporan las especies dentro de un grupo taxonómico en una región (VaneWright et al. 1991), o en comunidades (Arroyo \& Marañón 1990, Ojeda et al. 1995). La idea básica es que las especies contienen distinta cantidad de información que puede estar asociada a un diferente tiempo de existencia ("persistencia temporal" de Fiedler \& Ahouse 1991), diversidad filogenética (véase Bininda-Emonds et al. 2000 para una reciente actualización) o simplemente un variable número de congéneres (Ojeda et al. 1995). Como se ha indicado más arriba (Given 1994), aquí podemos considerar también como componente de interés para la conservación el valor científico de las especies o de sus poblaciones. Así, la existencia de caracteres indicativos de procesos ecológicos o evolutivos raros o que sirvan de casos de estudio apropiados para el análisis de éstos da valor a las especies y poblaciones que portan tales caracteres.

En las plantas, los caracteres relacionados con la reproducción tienen un significado evolutivo especial, tanto en procesos microevolutivos (en poblaciones) como macroevolutivos (asociados a especiación). En las angiospermas las flores presentan la mayor variabilidad e información por lo que sus caracteres son del mayor peso en taxonomía (e.g., Stebbins 1970). Sin embargo, en las plantas son también frecuentes los polimorfismos florales que no están necesariamente asociados a diferenciación taxonómica. En muchos casos el polimorfismo tiene una cierta base genética heredable y entonces puede ofrecer oportunidades para analizar si los procesos de selección natural son responsables o no de esas variaciones (véase Herrera 1996 para una revisión crítica).

Una clasificación sencilla de los polimorfismos florales los separa en continuos y discontinuos, con dos o más morfos florales en este caso. Los polimorfismos florales continuos han mostrado una diversidad de explicaciones posibles, no necesariamente adaptativas, como muestra Herrera (1996) para la longitud del tubo floral en un conjunto amplio de especies. Otra clasificación posible puede separar los polimorfismos del periantio de los que afectan a los órganos sexuales, y dentro de éstos los que afectan al género (diclinia o unisexualidad floral: dioecia, monoecia y situa- 
ciones relacionadas, revisado en Geber et al. 1999) o sólo a la posición de los órganos en la flor (heterostilia, polimorfismo estilar, enantiostilia, revisado en Barrett et al. 2000). Aunque estas dos clasificaciones de polimorfismo son independientes (Tabla 1) y se puedan dar muchas combinaciones, la mayoría de los casos con una buena base documental se refiere a los polimorfismos sexuales discontinuos.

Los polimorfismos florales han atraído a los naturalistas desde antiguo y han sido parte fundamental de la obra de Darwin, quien les dedicó una obra completa ("The different forms of flowers on plants of the same species", 1877), en la que dedicó seis capítulos (de un total de ocho) y casi un $80 \%$ de las páginas a la heterostilia (véase no obstante la revisión de Ornduff 1992). Sin embargo, todavía no se ha alcanzado un consenso completo en cuanto a los factores clave que determinan la aparición de la heterostilia, a pesar de la abundante literatura que ha generado a lo largo del siglo XX (Barrett 1992). Además, las especies heterostilas ofrecen una oportunidad magnífica para estudiar procesos relacionados con la función masculina vs. femenina en plantas hermafroditas y con la existencia de grupos morfofuncionales de cruzamiento ("assortative" versus "disassortative mating").

Narcissus es un género de plantas bulbosas mediterráneas que incluye unas 60-70 especies, la mayoría restringidas al Mediterráneo occidental. A pesar del evidente interés ornamental de estas plantas, que se evidencia por la existencia de sociedades de horticultura exclusivamente dedicadas a ellas y de numerosos textos técnicos y de divulgación para su cultivo, no existe una monografía taxonómica propiamente dicha (pero véase Blanchard 1990). Más sorprendentemente aún, la biología floral del grupo es escasamente conocida, con la excepción de $N$. longispathus (Herrera 1995) y N. tazetta (Arroyo \& Dafni 1995). En este grupo de plantas existe polimorfismo estilar y heterostilia con tal combinación de caracteres que ha modificado nuestra percepción del proceso evolutivo que ha conduci- do a la aparición de esta última. Recientemente se ha publicado una revisión de la situación de Narcissus con respecto a la heterostilia en la que se resaltaban estos hechos (Barrett et al. 1996). Sin embargo, posteriormente ha aparecido más información, en las mismas especies allí mencionadas o en otras nuevas, que ha aumentado y modificado el papel de este grupo de plantas en la explicación de un proceso que lleva más de un siglo en la agenda de los evolucionistas.

El objetivo de esta revisión es, por un lado, una puesta al día de los estudios de la heterostilia en Narcissus y su ajuste a hipótesis previas. Por otro lado, se usa la heterostilia para una valoración particular del género y algunas de sus especies para la conservación, de acuerdo con la singularidad de sus relaciones evolutivas y de su papel dilucidatorio de procesos evolutivos de mayor alcance. Para ello, se contrasta la información disponible para las distintas especies con las "listas rojas" existentes en el territorio donde se encuentra el mayor número de especies de Narcissus, la Península Ibérica y el noroeste de África.

\section{NARCISSUS Y LA HETEROSTILIA: UNA HISTORIA CONFLICTIVA}

\section{Modelos de evolución de la heterostilia}

La obra seminal de Darwin (1877) ya contiene un modelo cualitativo explícito de evolución de la heterostilia, aunque buena parte de la información empírica se refiere a Primula. El planteamiento básico es que la heterostilia es un mecanismo que promueve la polinización cruzada mediante el posicionamiento recíproco de anteras y estigma (y otros caracteres subordinados, véase Ganders 1979). El sistema de incompatibilidad dialélico (con dos grupos de cruzamiento) sería posterior y seleccionado para aumentar la eficacia en la transferencia legítima de polen (entre morfos); los ancestros deben ser o autocompatibles o incompatibles multialélicos. Este modelo ha

TABLA 1

Tipos de polimorfismos florales

Flower polymorphism types

\begin{tabular}{|c|c|c|}
\hline & Continuos & Discontinuos \\
\hline Sexuales & Varios & $\begin{array}{l}\text { De posición: heterostilia, } \\
\text { polimorfismo estilar, enantiostilia }\end{array}$ \\
\hline Periantio & Varios & De tamaño, color y olor \\
\hline
\end{tabular}


sido sucesivamente apoyado o retado en subsiguientes revisiones y propuestas, en las que se han incorporado datos empíricos de un gran número de especies (véase revisión en Barrett 1992 y Barrett et al. 2000). Los dos modelos recientes más influyentes son sin duda los de Charlesworth \& Charlesworth (1979) y de Lloyd \& Webb (1992a, 1992b), que plantean escenarios evolutivos contrapuestos. El primero niega los postulados básicos de Darwin y plantea como fuerza selectiva inicial la evitación de la depresión por consanguinidad, siendo el estadio morfológico inicial la homostilia (igual altura de anteras y estigmas; véase Fig. 3 en Barrett 1992, p. 11). Por el contrario, Lloyd \& Webb (1992a, 1992b) reformulan de forma explícita y cuantitativa la hipótesis de Darwin. Apoyándose en datos de historia natural y morfometría floral de un número de especies (Lloyd et al. 1990), estos autores proponen que el estado inicial es autocompatible y con separación de anteras y estigma en hercogamia de aproximación (estigma a mayor altura que anteras). La fuerza selectiva es el aumento de eficacia en la transferencia de polen y el siguiente paso la aparición de dimorfismo estilar: hay dos longitudes de estilo, pero la altura de las anteras virtualmente no varía. Este estadio debe ser inestable y, por tanto, raro en la naturaleza, debiendo conducir a la hercogamia recíproca o heterostilia por el incremento y disminución de la altura de los estambres. Más recientemente, Richards (1998) propone un tercer modelo más complejo y exclusivamente genético (véanse sus Fig. 1-3) en el que la carga genética y el ligamiento son los factores determinantes. En las Fig. 1, 2 y 4 de Richards (1998, pp. 77, 78 y 81) se incluyen esquemas de estos tres modelos cualitativamente diferentes, de los que evidentemente el más sencillo y con menos asunciones es el de Lloyd \& Webb (1992a, 1992 b), aunque esto no sea una medida estricta de parsimonia. Aunque todavía no existe una evaluación contrastada del ajuste de tales hipótesis, una serie de evidencias dan mayor apoyo a la hipótesis de Lloyd \& Webb (1992a, 1992b). El número de grupos taxonómicos diversos que apoyan la hipótesis de estos autores es también mayor y, aunque falta un análisis filogenético apropiado que establezca la condición ancestral (hipotéticamente hercogamia de aproximación, incompatibilidad multialélica o compatibilidad) en un número de grupos de plantas, hay cada vez más evidencias de que algunos grupos presentan un apoyo claro a esta hipótesis. De ellos, el más notable es Narcissus, en el que existen virtualmente todos los pasos requeridos por ese modelo: hercogamia de aproximación, dimorfismo estilar, distilia, tristilia, autocompatibilidad, incompati- bilidad multialélica (aunque es interesante que falte la dialélica; Dulberger 1964, Barrett et al. 1997).

Polimorfismos florales en Narcissus, una controversia recalcitrante

A pesar de la aparente escasez inicial de información detallada sobre biología y morfometría floral en Narcissus, este grupo de plantas había generado una abundante discusión previa a su significado teórico, que posteriormente ha arrojado mucha luz para dilucidar cuáles son los estados en los pasos requeridos en los modelos anteriormente mencionados. En Barrett et al. (1996, 1997) se detallan los pormenores de esta controversia entre A. Fernandes y A.J. Bateman y se aclara la situación del género y de $N$. triandrus en particular, la especie que fue objeto específico de esta discusión. El hecho clave mencionado y probado es que muchas de las especies que Fernandes describió como heterostilas son en realidad dimórficas para la longitud de estilo, algo que ya se citó para Narcissus tazetta (Dulberger 1964). Sin embargo, según Barrett et al. (1997), $N$. triandrus sí es heterostilo (tristilo), hecho que había sido puesto en duda por Bateman (1952) tras haberse descrito como tal muy tempranamente (Fernandes 1935). Más recientemente, Arroyo \& Barrett (2000) describen la existencia de distilia en $N$. albimarginatus. Es de resaltar que la asignación del carácter heterostilo está basada en la fundamentación de Lloyd \& Webb (1992a, 1992b), para los que el único requisito es la presencia de 2-3 morfos (distilia, tristilia) con posición recíproca de anteras y estigma (Tabla 2). Esta decisión está basada en el buen ajuste a las premisas del modelo de estos autores, más que en una definición ad hoc inconsistente; según otros modelos alternativos, en Narcissus no existiría la distilia desde el momento en que faltan otros caracteres supuestamente "necesarios" o previos al establecimiento de la reciprocidad estambres/ estigma, como la incompatibilidad dialélica.

Variación floral, polinización e hipótesis evolutivas en Narcissus

Pero en Narcissus hay más situaciones relevantes. De todos los grupos taxonómicos que se han citado en apoyo del modelo de Lloyd \& Webb (1992a, 1992b), probablemente es Narcissus el que en la actualidad sea más comprehensivo. Barrett et al. (1996) revisaron el papel de Narcissus en este contexto. Un breve repaso y algunos datos posteriores ponen de manifiesto la utilidad de 
TABLA 2

Modelos recientes para explicar la evolución de la heterostilia

Recent models for the evolution of heterostyly

\begin{tabular}{|c|c|c|c|}
\hline & $\begin{array}{c}\text { Charlesworth \& } \\
\text { Charlesworth (1979) }\end{array}$ & Lloyd \& Webb (1992) & Richards (1998) \\
\hline Fuerza selectiva & $\begin{array}{l}\text { Depresión por } \\
\text { consanguinidad }\end{array}$ & $\begin{array}{c}\text { Promoción "fitness" masculino } \\
\text { mediante una polinización } \\
\text { cruzada eficaz }\end{array}$ & Carga genética \\
\hline Supuestos genéticos & $\begin{array}{l}\text { Compatibilidad }-> \\
\text { incompatibilidad } 2 \\
\text { alelos }->\mathrm{n} \text { alelos }\end{array}$ & No necesarios & Ligamiento génico \\
\hline Evidencias & Staticeae (Plumbaginaceae) & $\begin{array}{c}\text { Anchusa, Lithodora, Narcissus, } \\
\text { Nivenia, Villarsia...* }\end{array}$ & $\begin{array}{l}\text { Primula sect. } \\
\text { Sphondylia }\end{array}$ \\
\hline Modelo cuantitativo & Sí & Sí & No \\
\hline Caracteres definitorios & $\begin{array}{c}\text { Hercogamia recíproca }+ \\
\text { inc. dialélica }+ \text { rasgos ancilares }\end{array}$ & Hercogamia recíproca & Dimorfismo estilar \\
\hline
\end{tabular}

*Anchusa: Schou \& Philipp (1984); Lithodora: comunicaciones personales de L. Navarro, J. Pannell, SCH Barrett y observaciones personales en distintas especies (cf. Valdés 1981); Narcissus: véase texto; Nivenia: evidencias indirectas a partir de Goldblatt \& Bernhardt (1990) y Goldblatt (1993), Villarsia: Ornduff (1988); véase Lloyd et al. (1990) y Lloyd \& Webb (1992a) para una evaluación de las evidencias taxonómicas

este género como sistema modelo para reconstruir la ruta evolutiva de la heterostilia. Adicionalmente, el género tiene algunas particularidades, como la relativa abundancia de dimorfismo estilar, que parece ser evolutivamente estable (Barrett et al. 2000). Esto no estaba previsto en el modelo inicial, en el que el dimorfismo estilar era considerado un paso necesario, pero inestable, en la evolución hacia la heterostilia.

En este género predomina el monomorfismo, tanto con hercogamia de aproximación (e.g. todas las especies de las Sects. Pseudonarcissi, Bulbocodii y Tapeinanthus) como sin hercogamia (Sects. Serotini, Narcissus). Otras secciones son mayoritariamente dimórficas para la longitud de estilo (e.g., Jonquilla, Apodanthae, Tazettae) aunque contienen algunas especies monomórficas sin hercogamia ( $N$. viridiflorus, $N$. marvieri, $N$. watieri, N. elegans). Finalmente, un par de especies de secciones diferentes son heterostilas: el ya mencionado $N$. triandrus (tristilo, en la monotípica Sect. Ganymedes) y N. albimarginatus (distilo, en la Sect. Apodanthae, véase más arriba). A pesar de ser este último un descubrimiento en sólo una especie geográficamente muy aislada, es un caso muy ilustrativo y ofrece vías nuevas de investigación sobre las rutas evolutivas posibles que han seguido estos polimorfismos florales. Se trata de una especie de morfología (Fig. 1), color y olor (al menos a los sentidos humanos, véase no obstante Dobson et al. 1997, para una compara- ción química cuantitativa de los olores de varias especies de Narcissus) muy parecidos al también heterostilo $N$. triandrus. El área de estas dos especies está separada unos $300 \mathrm{~km}$. Aunque no puede descartarse aún un ancestro común o un proceso de hibridación, esta separación y la posición taxonómica actual mutuamente aislada plantean la posibilidad de que esta semejanza floral se haya producido por convergencia (Arroyo \& Barrett 2000). Lamentablemente, existen pocos datos de insectos polinizadores en estas especies. En observaciones casuales $N$. triandrus es frecuentemente visitado por Anthophora pilipes y Bombus spp. (Barrett et al.1996), mientras que no hay ninguna observación disponible en $N$. albimarginatus. En su pariente $N$. cuatrecasasii, dimórfico, de olor, color y tubo floral similar, se observan con frecuencia y casi exclusivamente también Anthophora sp. visitando las flores (Arroyo, Dobson \& Kephart, datos inéditos)

De todos los caracteres florales, parece que la longitud y anchura del tubo floral pueden tener una importancia crítica. En las dos especies heterostilas el tubo es relativamente más corto y ancho que en las especies dimórficas (las especies monomórficas presentan una amplia variedad de tubos florales, véase Fig. 1). No existen muchos datos sobre insectos polinizadores en el conjunto de especies dimórficas, pero parece que son visitadas por un conjunto mucho más amplio de insectos, incluyendo abejas de diverso tama- 

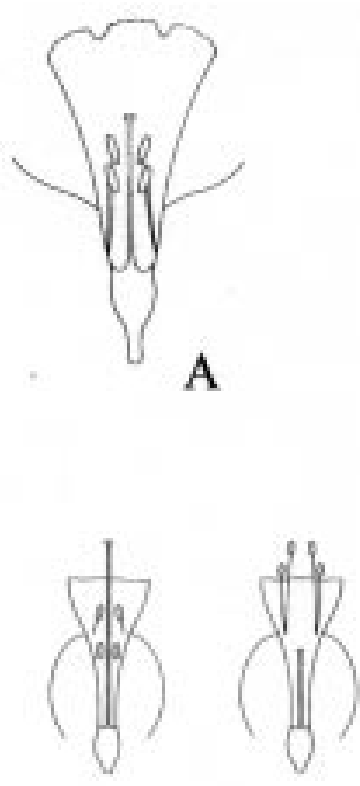

E
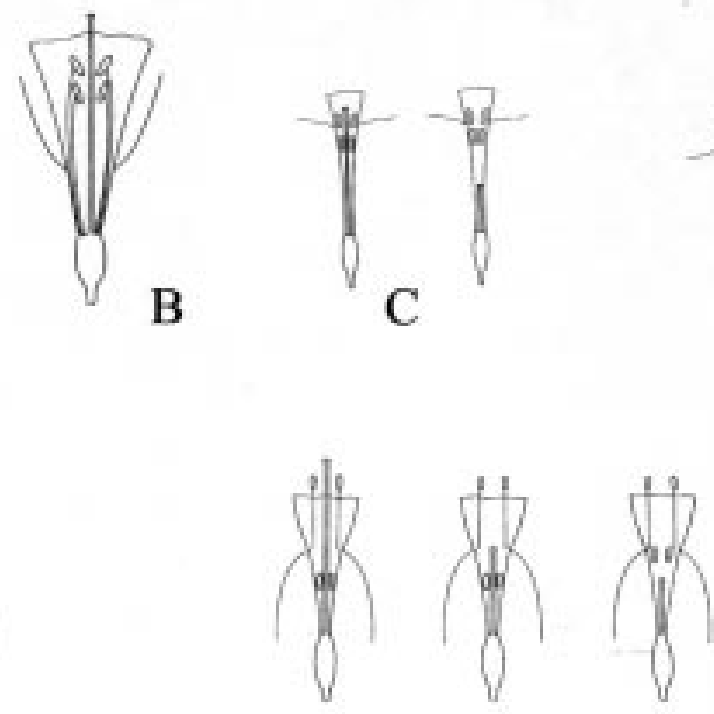

$\mathrm{F}$

Fig. 1: Representación esquemática de las morfologías florales de los tipos de polimorfismo encontrados en Narcissus. Se representan sólo dos de los tres estambres de cada verticilo para mayor claridad: (A) Narcissus bugei (sect. Pseudonarcissi); (B) N. cantabricus (sect. Bulbocodii); (C) N. assoanus (sect. Jonquillae); (D) N. cuatrecasasii (sect. Apodanthae); (E) N. albimarginatus (sect. Apodanthae; (F) N. triandrus (sect. Ganymedes).

Schematic drawing of the flower morphology of the polymorphisms found in Narcissus. For simplicity, only two out of the three stamens in each whorl are represented: (A) Narcissus bugei (sect. Pseudonarcissi); (B) N. cantabricus (sect. Bulbocodii); (C) N. assoanus (sect. Jonquillae); (D) N. cuatrecasasii (sect. Apodanthae); (E) N. albimarginatus (sect. Apodanthae; (F) N. triandrus (sect. Ganymedes).

ño, lepidópteros diurnos y nocturnos, sírfidos y bombílidos entre otros (Arroyo \& Dafni 1995 para N. tazetta, Arroyo, Dobson \& Kephart resultados no publicados para $N$. assoanus). Barrett et al. (1996) y Arroyo \& Barrett (2000) sugirieron que la estrechez del tubo floral debe suponer una restricción morfológica importante que ha impedido o dificultado el posicionamiento recíproco de los órganos sexuales típico de la heterostilia a partir del dimorfismo estilar, a pesar de las predicciones del modelo de Lloyd \& Webb (1992a, 1992b). La morfología floral de N. albimarginatus y N. triandrus apoya esta sugerencia. Como ya hicieron notar Barrett et al. (1996) y Arroyo \& Barrett (2000), las especies dimórficas no varían sólo en la longitud del estilo. Sin llegar a presentar una situación recíproca, las anteras (particularmente el verticilo inferior de los dos existentes en Narcissus, Fig. 1) alcanzan alturas ligera pero significativamente diferentes en los dos morfos. Un análisis morfométrico preliminar muestra que esta diferencia es mayor precisamente en especies dimórficas de la Sect. Apodanthae, particularmente en N. cuatrecasasii (Tabla 3).
Evidentemente, sería necesario disponer de una filogenia robusta del género Narcissus para probar esta hipótesis; esto es, que la heterostilia aparece asociada a una morfología floral particular y que ello ocurre de forma independiente en distintas especies. Mientras que la elaboración de una filogenia completa es tediosa por un número de problemas entre los que se encuentran la cantidad de especies, su confuso estatus taxonómico y una importante reticulación por el alto nivel de hibridación (Fernandes 1975), una solución puede venir dada por el análisis filogenético de la Sect. Apodanthae. Esta sección, bien definida, está compuesta por siete especies que muestran un claro patrón geográfico y, según se desprende de la descripción hecha anteriormente, una variedad amplia de condiciones de polimorfismo (Fig. 2). En gran medida este grupo de especies puede servir como una submuestra que permite estudiar, no ya todos los pasos del modelo de Lloyd \& Webb (1992a, 1992b), sino también el proceso particular de evolución de la distilia a partir del dimorfismo estilar y, secundariamente, la reversión al monomorfismo, representado éste por $N$. marvieri y $N$. watieri (J. Arroyo resultados no 
TABLA 3

Separación entre las anteras de los verticilos superior e inferior en los dos morfos de longitud de estilo en varias especies dimórficas de Narcissus (datos reanalizados a partir de Barrett et al. 1996 y Arroyo \& Barrett 2000). Las especies están ordenadas de menor a mayor valor de la diferencia entre morfos

\begin{abstract}
Distance (in mm) between upper-and lower-whorl anthers in both style-length morphs of several dimorphic Narcissus species (re-analysed data after Barrett et al. 1996 and Arroyo \& Barrett 2000). Species are arranged in increasing value of between-morph difference
\end{abstract}

\begin{tabular}{|c|c|c|c|}
\hline & \multicolumn{3}{|c|}{ Separación entre anteras superiores e inferiores $(\mathrm{mm})$} \\
\hline & Morfo de estilo largo & Morfo de estilo corto & Diferencia entre morfos \\
\hline N. papyraceus & 4,61 & 3,65 & 0,96 \\
\hline N. assoanus & 4,57 & 3,44 & 1,13 \\
\hline N. rupicola & 5,73 & 4,42 & 1,31 \\
\hline N. gaditanus & 5,06 & 3,55 & 1,51 \\
\hline N. calcicola & 4,68 & 2,80 & 1,88 \\
\hline N. cuatrecasasii & 4,49 & 2,18 & 2,31 \\
\hline N. albimarginatus & 6,87 & 2,12 & 4,75 \\
\hline
\end{tabular}

publicados) en el extremo meridional del ámbito geográfico del género (Alto Atlas, Marruecos). Es interesante que el tubo floral de estas dos especies sea el más largo y estrecho de toda la sección.

Una filogenia molecular robusta de la sección permitiría someter a prueba la hipótesis de que la especie distila $N$. albimarginatus forme un par hermano con N.cuatrecasasii (la especie que, sin ser heterostila, presenta la mayor diferencia entre morfos en la separación entre anteras y estigmas, Tabla 3 y Fig. 1). Estas dos especies son además las más cercanas geográficamente. Sería necesario representar la separación relativa entre anteras y estigmas (grado de hercogamia recíproca) en el cladograma. Dada la aparente congruencia geográfica de esta hipótesis (Fig. 2), sería posible dar un segundo paso al nivel de población. Un análisis filogeográfico de las poblaciones de $N$. cuatrecasasii (y la única conocida de $N$. albimarginatus) podría revelar un patrón de diferenciación más fino y su relación con las diferencias en la separación anteras-estigma entre poblaciones. La población de $N$. cuatrecasasii recientemente citada en el norte de Marruecos (Benabid 2000) a unos $20 \mathrm{~km}$ de $N$. albimarginatus es especialmente interesante en este contexto.

\section{Dimorfismo estilar estable taxonómicamente pero inestable ecológicamente}

Aún existiendo distilia en Narcissus, el dimorfismo estilar sigue siendo el polimorfismo floral dominante en el género, con lo que las cuestiones planteadas sobre su estabilidad evolutiva siguen

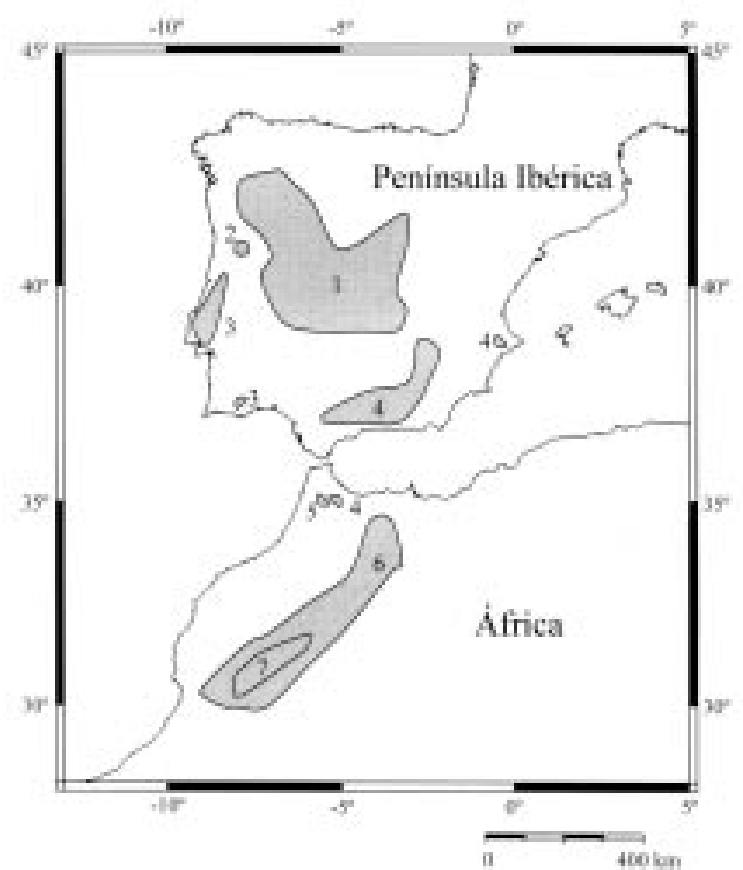

Fig. 2: Distribución geográfica de la sección Apodanthae del género Narcissus. Elaborado a partir de Blanchard (1990), Moreno-Saiz \& SainzOllero (1992) y Benabid (2000): (1) N. rupicola; (2) N. scaberulus; (3) N. calcicola; (4) N. cuatrecasasii; (5) N. albimarginatus; (6) $N$. marvieri; (7) N. watieri.

Geographic range of the section Apodanthae in the genus Narcissus. Elaborated after Blanchard (1990), Moreno-Saiz \& Sainz-Ollero (1992) and Benabid (2000): (1) N. rupicola; (2) N. scaberulus; (3) N. calcicola; (4) N. cuatrecasasii; (5) N. albimarginatus; (6) N. marvieri; (7) $N$. watieri. 
estando vigentes (cf. Barrett et al. 1996). Esta situación contrasta fuertemente con lo encontrado al nivel de población en las especies dimórficas. Casi todas las poblaciones están dominadas por el morfo de estilo largo, llegando en ocasiones a ser monomórficas para éste (Barrett et al. 1996, Baker et al. 2000a, 2000b). Sin embargo, casi nunca ha aparecido dominancia del morfo corto (excepto en alguna población aislada de $N$. papyraceus, J. Arroyo et al. resultados no publicados) y nunca monomorfismo para éste. Las especies dimórficas en las que se ha examinado un número alto de poblaciones muestran además variaciones en la dominancia del morfo largo (Tabla 4). En algunos casos (N. assoanus: Baker et al. 2000a, $N$. papyraceus: Arroyo et al. resultados no publicados) existe incluso un patrón ecogeográfico claro en relación con el aislamiento y adecuación de los hábitats propicios para estas especies. De hecho, esta variación en la proporción de morfos está asociada al tamaño de las poblaciones, de forma que en las mayores es más probable encontrar proporciones iguales de morfos (isopletia). La distribución del tamaño de las poblaciones está agregada en algunas zonas geográficas en ambas especies. En N. cuatrecasasii la proporción variable de morfos también tiene un patrón geográfico (J. Arroyo resultados no publicados), aunque no parece ser gradual: la menor proporción de plantas de morfo largo se encuentra en la Sierra de Cazorla (Fig. 2), incluso con poblaciones isopléticas $($ media $=0,62)$, mientras que en la cercana Sierra de Mágina esta proporción es mayor $(0,85)$. En el extremo occidental del área de distribución de esta especie (Sierra de Grazalema) la proporción es intermedia $(0,72)$; en ninguna de las zonas se ha observado una correlación entre tamaño de población y proporción de morfos. En $N$. dubius no se ha encontrado ningún tipo de patrón ecogeográfico (Baker et al. 2000a).
Modelos que predicen la proporción de morfos en poblaciones de especies dimórficas

Varios hechos pueden ayudar a comprender la variación y dominancia del morfo largo. En primer lugar, el tipo de sistema genético de incompatibilidad presente permite el cruzamiento entre y dentro de morfos pero los individuos son autoincompatibles (Dulberger 1964, Barrett et al. 1977, 1996, Sage et al. 1999, Arroyo et al. resultados no publicados). Todas las especies dimórficas mencionadas más arriba (excepto la autocompatible N. dubius, Baker et al. 2000b) tienen este sistema, lo que apoya explícitamente la hipótesis de Lloyd \& Webb (1992a,b). Esto también explica que las poblaciones monomórficas puedan mantenerse mediante la producción de semillas. Además, hay cierta evidencia de que la herencia de la longitud del estilo está gobernada por un locus con dos alelos y dominancia ( $s s$ estilo largo, Ss estilo corto, Dulberger 1964). Entre las diversas consecuencias que esto puede tener es de notar que una población fundada sólo por plantas de estilo largo permanecerá monomórfica mientras no inmigre alguna planta corta o aparezca el alelo alternativo por mutación. Una población fundada por plantas de estilo corto segregará en las siguientes generaciones ambos morfos. En segundo lugar, la altura de las anteras no es exactamente igual en los dos morfos, de forma que el morfo corto presenta mayor separación estigma-anteras que el largo, es decir, los estigmas del morfo corto alcanzan una altura más parecida al verticilo inferior de anteras del morfo opuesto, en comparación con el morfo largo (Fig. 1). Siguiendo el razonamiento de Lloyd \& Webb (1992a, 1992b) en cuanto a la mayor probabilidad de transferencia de polen entre anteras y estigmas de similar altura, esto es, de distintos morfos en especies heterostilas ("disassortative mating"),

TABLA 4

Proporción de morfo de estilo largo en especies de Narcissus con dimorfismo estilar

Proportion of the long-style morph in style dimorphic Narcissus species

\begin{tabular}{lcccc}
\hline Especie & Proporción de plantas del morfo de estilo largo & Referencia \\
\cline { 2 - 4 } & Media & Rango & $\begin{array}{c}\text { Número de } \\
\text { poblaciones } \\
\text { examinadas }\end{array}$ \\
\hline Narcissus assoanus & 0,59 & $0,46-0,93$ & 46 & Baker et al. (2000) y datos inéditos \\
Narcissus dubius & 0,9 & $0,77-1,0$ & 21 & Baker et al. (2000) y datos inéditos \\
Narcissus papyraceus & 0,88 & $0,37-1,0$ & 68 & J. Arroyo et al. resultados no publicados \\
Narcissus cuatrecasasii & 0,73 & $0,42-0,99$ & 21 & J. Arroyo resultados no publicados \\
\hline
\end{tabular}


debemos deducir que en las especies dimórficas de Narcissus el morfo corto debe sufrir mayores niveles de polinización desde el morfo opuesto (polinización legítima), mientras que en el morfo largo el grado de polinización desde el mismo (polinización ilegítima) debe ser mucho mayor. Finalmente, el sitio exacto de acción del mecanismo de incompatibilidad permanece desconocido, aunque actúa al nivel del ovario y cuando la polinización es con polen propio, deja los óvulos inútiles, de forma similar a los mecanismos postzigóticos (Dulberger 1964, Sage et al. 1999). Si hay un nivel alto de autopolinización (incluyendo geitonogamia), ésta debe determinar un "descuento de óvulos" disponibles para la reproducción y se ha propuesto un modelo (Barrett et al. 1996) en el que la tasa de descuento es diferente entre morfos. Sin embargo, no se ha observado que la producción de semillas sea muy diferente entre morfos en las especies analizadas (Baker et al. 2000b, J. Arroyo et al. resultados no publicados). Por ello, el descuento por sí solo no es suficiente para explicar la sobredominacia del morfo largo. En cambio, Baker et al. (2000b) han elaborado un modelo genético de cruzamiento que pone más énfasis en la tasa relativa de cruzamiento dentro y entre morfos y calcula la frecuencia de cada genotipo de morfo en equilibrio en función de la tasa relativa de cruzamiento entre/ dentro de morfos.

Hipotéticamente, el morfo largo debe sufrir mayor cantidad de cruzamiento con otras plantas del mismo tipo en comparación con el morfo corto. Esta predicción podría ser fácilmente puesta a prueba si las especies dimórficas de Narcissus presentaran, como la mayoría de las heterostilas, dimorfismo de polen (tamaño y/o morfología, véase por ejemplo Ganders 1979). De hecho en la literatura hay abundantes ejemplos de estudios de transferencia de polen en los morfos de especies heterostilas usando esta facilidad (véase revisión en Dulberger 1992). Hasta ahora no se ha encontrado este dimorfismo polínico en Narcissus (Barrett et al. 1996) lo que dificulta la tarea. Una aproximación, que estamos llevando a cabo en la actualidad, consiste en la estimación de estas cantidades de transferencia de polen en poblaciones experimentales: monomórficas largas, dimórficas y monomórficas cortas. La aproximación es experimental desde el momento en que las últimas no existen en la naturaleza, pero también para evitar el efecto inherente del área en poblaciones naturales, que están a menudo muy segregadas espacialmente y la fenología y los polinizadores pueden ser diferentes. Si el modelo de Baker et al (2000b) es correcto, debe esperarse que la cantidad de polen depositado en los estig- mas de las plantas de poblaciones monomórficas largas y dimórficas sea aproximadamente igual y mucho más baja en las monomórficas cortas. Un paso experimental adicional está consistiendo en la emasculación de las flores para poder conocer la cantidad absoluta de transferencia de polen en los morfos desde plantas de un morfo hasta flores receptoras emasculadas de ambos morfos. Finalmente, este experimento se está repitiendo en especies dimórficas con diferente grado de hercogamia (N. papyraceus y $N$. assoanus -bajay N. cuatrecasasii-alta-) ya que ésta debe afectar directamente el grado de cruzamiento entre morfos. Como control se replica el experimento en una especie heterostila ( $N$. triandrus), en la que la polinización legítima debe ser relativamente mayor en todos los morfos, aunque difiera entre éstos (cf. Dulberger 1992).

Como consecuencia de los patrones mostrados y los modelos explicativos mencionados se puede concluir que el género Narcissus constituye un caso de estudio excepcional para probar una hipótesis evolutiva de carácter muy general, sobre la evolución de la heterostilia. Ésta ofrece oportunidades únicas para estudiar la evolución de la fertilización cruzada. De hecho, la heterostilia aparece tradicionalmente explicada en detalle como ejemplo de estudio en textos de genética, incluso los más básicos (e.g., Hartl 1988). La aparente contradicción entre las hipótesis existentes hace que todavía hoy siga siendo un tópico "caliente" para los biólogos evolucionistas (véase Richards 1997, 1998). En este contexto, Narcissus puede ofrecer mucha información dilucidatoria en cuanto al establecimiento de este polimorfismo en asociación al proceso de especiación en el género. La sección Apodanthae de este género puede constituir un "núcleo" donde se concentra una alta diversidad, no de especies (siete) sino de estrategias evolutivas (tres condiciones de polimorfismo). Más recientemente, se ha comprobado que algunas especies presentan variaciones poblacionales con patrones interesantes que permiten probar hipótesis ecológicas y microevolutivas sobre las condiciones necesarias para el mantenimiento del polimorfismo.

\section{NARCISSUS Y LA CONSERVACIÓN DE LA FLORA Y DE LA DIVERSIDAD}

\section{Las listas rojas de especies amenazadas y en peligro}

En la práctica diaria de la conservación biológica, ésta debe ser aplicada a objetos: especies o áreas geográficas. En el primer caso, esto se plasma en 
el establecimiento de listas de especies amenazadas ("listas rojas"), con reglamentación legal sobre su posible uso, incluyendo la prohibición total de cualquier actividad más allá de la propia conservación; la aparición de especies de Narcissus en las listas rojas es muy frecuente. En el segundo caso, las áreas geográficas tienen frecuentemente interés de conservación por su contenido en especies, bien en términos cuantitativos (número de especies) o cualitativos (interés particular de algunas especies). Por otro lado, la conservación se enfrenta casi siempre a la limitación de recursos económicos disponibles, de forma que rara vez se pretende conservar toda la lista de especies de un territorio amplio, es decir, una flora o una fauna regional con entidad biogeográfica. Por ello, uno de los objetivos de la biología de la conservación es detectar áreas y grupos taxonómicos que contengan la mayor diversidad posible para concentrar en ellos los recursos disponibles. Los patrones y procesos que afectan a la diversidad biológica son temas recurrentes en ecología y biogeografía, si bien en biología de la conservación, debido a las limitaciones prácticas, la diversidad se ha tratado con frecuencia como riqueza de especies. Los sitios muy ricos en especies han tenido mayor probabilidad de ser considerados objetos de conservación. Recientemente, algunos autores (Pressey et al. 1993, Williams \& Humphries 1993) han considerado que el número absoluto de especies es un criterio que puede conducir a un aprovechamiento subóptimo de los recursos, por lo que consideran que las áreas a conservar deben complementarse en el número e identidad de especies contenidas. En la Península Ibérica, Castro et al. (1996) han utilizado esta aproximación para proponer y categorizar una serie de áreas de conservación de plantas que sumadas reúnan el mayor número de especies. Muchas de estas áreas contienen especies de Narcissus características de ellas. En algunos casos incluso se ha propuesto una atención especial en función de la exclusividad de especies de Narcissus (e.g., N. scaberulus o N. cavanillesii en algunas pequeñas zonas del centro y sur de Portugal, respectivamente (A. Rosseló y B. García comunicación personal).

Criterios para la inclusión de especies en las listas rojas

Dado que Narcissus es un género muy diversificado y con una concentración de especies en el oeste del Mediterráneo, donde muchas son endémicas, es de esperar que su presencia en las listas rojas de esa zona sea frecuente. Si a ello se añade el interés intrínseco de Narcissus como sistema modelo para estudiar procesos evolutivos de alcance general, es lógico considerar este grupo de plantas idóneo para comprobar si su tratamiento consigue recoger la diversidad e información biológica de forma equilibrada. Para ello se han seleccionado cuatro fuentes de información publicadas que son, explícita o implícitamente, listas rojas comprehensivas de la flora de algunas de las zonas con mayor concentración de especies de este género: España con (VV.AA. 2000) y sin Canarias (Gómez-Campo 1987), Andalucía (Blanca et al. 1999a, 1999b) y Marruecos (Fennane \& Ibn-Tattou 1998). En la Tabla 5 pueden comprobarse algunos datos básicos sobre los números de especies vasculares y de Narcissus en particular en estas listas.

A pesar de las ventajas que este género reporta, también hay algunos inconvenientes metodológicos. El principal es la falta de una monografía taxonómica actualizada que permita un tratamiento homogéneo en cualquier análisis evolutivo o de conservación. Por ello, se han usado floras estándar de las regiones consideradas para el cálculo de los números totales de especies, sin entrar en valoraciones del estatus taxonómico, que no es objetivo de este trabajo. Por la misma razón, se ha respetado la asignación taxonómica dada en cada lista roja. En segundo lugar, muchas de las especies de Narcissus han estado sujetas a cultivo ornamental desde hace siglos, sometidas a selección artificial y con frecuencia escapadas. Cuando hay evidencia de esto en alguna región, las especies afectadas no son tenidas en cuenta para ella. Finalmente, dada la alta hibridación aparente en este género, tampoco se han tenido en cuenta los híbridos cuando son definidos claramente como tales, pues inflaría sobremanera el número total de especies.

¿Qué debe tener una especie para que aparezca en las listas rojas? Los criterios declarados en estas obras no son exactamente iguales pero todos tienen el objetivo de incluir las especies consideradas amenazadas en diverso grado, constituyendo las distintas categorías niveles de vulnerabilidad. En todas estas listas se reconoce la utilidad de los criterios de UICN, siguiéndose en mayor (e.g., VV.AA. 2000) o menor (Fennane \& Ibn Tattou 1998) medida. En la práctica casi todos los criterios son básicamente geográficos (i.e., distribución total o parcialmente restringida) y/o ecológicos (supuesta vulnerabilidad a cambios ambientales, antrópicos o no). Existen también diferencias en cuanto al número de categorías establecidas, aunque para este análisis se han agrupado en dos, con objeto de homogenizar el tratamiento. Aunque en ningún caso se considera 
explícitamente la rareza taxonómica o filogenética, a veces se ha considerado la estabilidad taxonómica (véase Gómez-Campo 1987), procurando categorías uniformes (especies o subespecies). En ningún caso aparece el interés científico per se de las especies como un criterio general, aunque en ocasiones se hacen comentarios al respecto (e.g., N. calcicarpetanus, GómezCampo 1987). Las grandes variaciones en el número total de taxones incluidos en las listas (Tabla 5) se deben a la superficie del área, la categoría taxonómica considerada y la consideración adicional del endemismo como un valor intrínseco merecedor de conservación, independientemente de la rareza en el territorio (explícitamente en Fennane \& Ibn-Tattou 1998, e implícitamente en España, al considerar la gran cantidad de especies endémicas en Canarias, VV.AA. 2000).

En todas estas listas aparece representado Narcissus, oscilando entre 6 y 18 especies o subespecies. De hecho Narcissus debe tener cierto atractivo incluso estético dado que en dos de las obras aparece representado en la portada: como única planta en Gómez-Campo (1987) y compartiendo cartel con otras 14 plantas en Blanca et al. (1999a, 1999b). Al analizar las listas de especies de Narcissus lo primero que llama la atención es su distribución taxonómica. Si consideramos la categoría taxonómica de sección, relativamente estable a pesar de la confusa taxonomía del género, se observa que hay dos que están más representadas que el resto (Tabla 6): Pseudonarcissi en España y
Bulbocodii en Marruecos. La primera no pasa al sur del Estrecho de Gibraltar, mientras que la segunda está muy diversificada en ambas zonas. Este sesgo taxonómico impone una gran restricción en los tipos morfológicos representados. Este planteamiento está basado en una valoración taxonómica intrínsecamente equivalente de todas las especies, de forma que sólo su distribución o la amenaza de su extinción parecen ser valoradas. Sin embargo, hay ya un cuerpo de conocimiento teórico que propone que cuando se elaboran listas de especies a proteger, debe incorporarse el criterio de recoger la mayor diversidad evolutiva y filogenética posible (May 1990, Vane-Wright et al. 1991). Sorprendentemente, este tipo de información no es considerado en textos influyentes de biología de la conservación (Primack 1993, Given 1994); sólo Fiedler \& Ahouse (1992) consideran algo que está relacionado con la singularidad filogenética, la persistencia temporal o edad de las especies, aunque su estimación cuantitativa está muy limitada. Obviamente, para calcular la diversidad filogenética es necesario disponer de una filogenia bien resuelta, que todavía no está disponible para Narcissus (S.C.H. Barrett comunicación personal). Tampoco pueden usarse las propuestas evolutivas de Fernandes (1975) para este género, por no emplear un análisis filogenético propiamente dicho y por utilizar principalmente datos cariológicos que sólo permiten delinear relaciones de parentesco en aquellos conjuntos de especies con más variación en el número cromosómico.

TABLA 5

Número de taxones (especies o subespecies de Narcissus) incluidos en diversas listas rojas del Mediterráneo occidental

Number of taxa (species or subespecies) in the genus Narcissus included in several red lists of the western Mediterranean

\begin{tabular}{|c|c|c|c|c|c|}
\hline Área geográfica & $\begin{array}{l}\text { Número de } \\
\text { taxones } \\
\text { vasculares }\end{array}$ & $\begin{array}{l}\text { Proporción del } \\
\text { total de } \\
\text { la flora }(\%)\end{array}$ & $\begin{array}{l}\text { Número de } \\
\text { taxones } \\
\text { de Narcissus }\end{array}$ & $\begin{array}{c}\text { Proporción } \\
\text { del total } \\
\text { del género }(\%)\end{array}$ & Fuente \\
\hline $\begin{array}{l}\text { España peninsular } \\
\text { y Baleares }\end{array}$ & 3001 & 5 & 6 & $14,3^{2}$ & Gómez-Campo (1987) \\
\hline España & $1.414^{3}$ & 19 & $9(18)^{6}$ & $21,4^{2}$ & VVAA (2000) \\
\hline Andalucía & 1913 & 5 & 6 & $33,3^{4}$ & $\begin{array}{l}\text { Blanca et al. (1999a, } \\
\text { 1999b) }\end{array}$ \\
\hline Marruecos & $2.819^{3}$ & 59,5 & $10(12)^{7}$ & $45,5^{5}$ & $\begin{array}{l}\text { Fennane \& Ibn-Tattou } \\
\text { (1998) }\end{array}$ \\
\hline
\end{tabular}

${ }^{1}$ Incluye sólo especies

${ }^{2}$ Sobre un total de 42, promediando los datos de Blanchard (1990) y Webb (1980)

${ }^{3}$ Especies y subespecies

${ }^{4}$ Sobre un total de 18, a partir de Valdés (1987) y Diosdado-Fernández \& Pastor-Díaz (1997)

${ }^{5}$ Sobre un total de 22, a partir de Maire (1959)

${ }^{6}$ Entre paréntesis, número de especies incluyendo aquéllas de las que no se dispone de datos suficientes

${ }^{7}$ Entre paréntesis, número de especies incluyendo aquéllas endémicas pero no raras 
Número de especies y subespecies de Narcissus en las listas rojas de España y Marruecos y en las floras correspondientes; se ha seguido la separación en secciones recogida en RHS (1998)

Number of species and subespecies in the genus Narcissus included in red lists and floras of Spain and Morocco; taxonomy follows section delimitation of RHS (1998)

\begin{tabular}{|c|c|c|c|c|c|c|c|}
\hline \multirow[t]{2}{*}{ Sección } & \multicolumn{2}{|c|}{$\begin{array}{l}\text { Número en } \\
\text { España }\end{array}$} & \multicolumn{2}{|c|}{$\begin{array}{l}\text { Número en la lista } \\
\text { roja de España }\end{array}$} & \multicolumn{2}{|c|}{$\begin{array}{l}\text { Número en } \\
\text { Marruecos }\end{array}$} & \multirow{2}{*}{$\begin{array}{c}\text { Número en la lista } \\
\text { roja de Marruecos }\end{array}$} \\
\hline & $\begin{array}{c}\text { Blanchard } \\
\text { (1990) }\end{array}$ & $\begin{array}{l}\text { Webb } \\
(1980)\end{array}$ & $\begin{array}{c}\text { Gómez-Campo } \\
\text { (1987) }\end{array}$ & $\begin{array}{l}\text { VVAA } \\
(2000)\end{array}$ & $\begin{array}{l}\text { Blanchard } \\
\text { (1990) }\end{array}$ & $\begin{array}{l}\text { Maire } \\
(1959)\end{array}$ & \\
\hline Tapeinanthus & 1 & 1 & 0 & 0 & 1 & 1 & 0 \\
\hline Serotini & 1 & 1 & 0 & 0 & 1 & 11 & 0 \\
\hline Aurelia & 0 & 0 & 0 & 0 & 1 & 1 & 1 \\
\hline Tazettae & 5 & 4 & 1 & 1 & 5 & 71 & 1 \\
\hline Narcissus & 2 & 1 & 0 & 0 & 0 & 0 & 0 \\
\hline Jonquillae & 8 & 6 & 1 & 1 & 1 & 11 & 0 \\
\hline Apodanthae & 2 & 21 & 0 & 0 & 3 & 21 & $4^{2}$ \\
\hline Ganymedes & 1 & 2 & 0 & 0 & 0 & 0 & 0 \\
\hline Bulbocodii & 5 & 4 & 0 & 2 & 8 & 8 & 6 \\
\hline Pseudonarcissi & 20 & 13 & 4 & 14 & 0 & 0 & 0 \\
\hline
\end{tabular}

${ }^{1}$ La sección Apodanthae es considerada dentro de la sección Jonquillae por Webb (1980); las secciones Serotini, Tazettae y Jonquillae son consideradas dentro de la sección Hermione y la sección Apodanthae dentro de la sección Queltia por Maire (1959) pero se ha separado para mayor claridad y homogeneidad

${ }^{2}$ El número de especies amenazadas es mayor que el total de especies debido al descubrimiento reciente de $N$. albimarginatus y de $N$. cuatrecasasii

La concentración taxonómica de especies de Narcissus en las listas rojas muestra además un efecto que resulta llamativo en cuanto a la morfología floral. La mayor parte de las especies incluidas son monomórficas (secciones Pseudonarcissi y Bulbocodii) y muy parecidas entre sí en comparación con el resto. Ambas secciones representan sólo la condición ancestral (hercogamia de aproximación) en el modelo de Lloyd \& Webb (1992a, 1992b). Sin embargo, son muy homogéneas también en tamaño floral, tanto absoluto como relativo (en proporción al tamaño de la planta) y las flores son casi siempre solitarias. Podría significar ello un mayor poder de "atracción" al ojo humano que se refleja en que por término medio, las flores de las especies amenazadas tienen flores más grandes (Tabla 7), con la excepción del tamaño relativo en las especies no amenazadas de Andalucía. No obstante, éste es un análisis muy preliminar, sin corrección del efecto filogenético propiamente dicho, al faltar la filogenia. Ni siquiera es posible utilizar pares de especies en cada sección para realizar unos sencillos contrastes filogenéticamente independientes (PICs, en el sentido de Westoby et al. 1996), al no existir un número suficiente de secciones taxonómicas con especies amenazadas y no amenazadas para comparar sus tamaños florales. Sin embargo, sí merece destacarse que el patrón es muy parecido en Andalucía y en Marruecos
(Tabla 7), a pesar de que la representación de las secciones es muy diferente (véase más arriba). Todo ello apunta a que está afectando un sesgo frecuentemente no reconocido pero documentado en contextos conservacionistas, que relaciona positivamente el tamaño del organismo y su eco conservacionista (Herrera 1989). Con el mismo razonamiento podría aplicarse esto a órganos cuando éstos tienen especial poder de atracción (e.g., flores). No en vano la mayoría de los cultivares ornamentales de Narcissus derivan de especies de la sección Pseudonarcissi (Brickell 1994). Sería interesante realizar un análisis del tamaño floral en relación al estatus de conservación en otros grupos más apropiados metodológicamente. No obstante hay que destacar un posible efecto práctico positivo de este sesgo. Una planta vistosa, de flores grandes, o propia de un lugar prístino, tiene más posibilidades de generar interés general de conservación. De forma no tan inconsciente, los gestores de conservación suelen aprovechar este sesgo psicológico para obtener más recursos económicos para la conservación.

\section{Información biológica en las listas rojas}

Dado que la tradición de elaboración de listas rojas es reciente en el área geográfica en conside- 
ración, es importante que se lleve a la práctica incluso con escasez de conocimientos sobre la biología de las plantas incluidas. Sin embargo, es conveniente que se recopile la información existente sobre ellas y, aunque la importancia ecológica o evolutiva de las especies no pueda considerarse como criterio general y homogéneo para todos los grupos, al menos puede ser tenida en cuenta en el futuro. Además de la información taxonómica y evolutiva, las especies contienen otros tipos de información también importantes en biología de la conservación y tradicionalmente admitidas en las propuestas teóricas: las relaciones ecológicas con otras especies. Se definen así las llamadas especies clave ("key stone species"), debido a que su desaparición puede provocar una reacción en cadena de efectos negativos para la conservación de otras especies (Primack 1993, Given 1994). La incorporación de esta información ecológica como criterio de selección para las especies candidatas a las listas rojas está muy limitada. Para ello sería deseable disponer de un archivo de datos similar al incluido en la serie "Biological flora of the British Isles", que aparece regularmente en "Journal of Ecology" (hasta el presente incluye algo más de dos centenares de especies, aproximadamente un $15 \%$ de la flora de ese territorio). Sin embargo, esto está muy limitado en una zona donde la diversidad florística es aproximadamente diez veces superior. En Europa central, donde la diversidad florística es relativa- mente alta, se ha comenzado recientemente una serie similar (Matthies \& Poschold 2000).

En dos de las listas rojas objeto de análisis se incluye información sobre las especies (GómezCampo 1987, Blanca et al. 1999a, 1999b). Las dos restantes (Fennane \& Ibn-Tattou 1998, VV.AA. 2000) pretenden ser sólo listas, son muy recientes y es de esperar que en un futuro próximo las especies incluidas sean objeto de estudios detallados. En Gómez-Campo (1987) la información era necesariamente muy concisa, poniéndose casi todo el énfasis en la distribución geográfica. En Blanca et al. (1999a, 1999b) se incluye una ficha documental de cada especie con abundantes datos sobre aspectos de su biología relevantes para la conservación. Parte de los datos han sido obtenidos ad hoc para la obra y otros provienen de referencias bibliográficas. Dado que ésta es la obra que aporta mayor cantidad de datos por especie incluida, puede resultar interesante como fuente de información sobre características de las poblaciones/especies relevantes para los patrones evolutivos que más arriba se han explicado. En particular, los datos más necesarios son los referentes a biología floral y sistema de reproducción, las fuerzas potencialmente conductoras en los distintos modelos de evolución de la heterostilia. Un breve repaso a los datos aportados para Narcissus revela una situación desigual.

La única especie estudiada a fondo en un estudio previo es N. longispathus (Herrera 1995) de la que

TABLA 7

Tamaño de la flor (media \pm desviación típica) en especies de Narcissus con diversa consideración de amenaza en las listas rojas. Los valores para cada especie se han obtenido a partir de los datos incluidos en floras estándar de las regiones consideradas. Cuando los valores se proporcionan como intervalos, se toma la media entre los extremos

Flower size (mean \pm standard deviation) of Narcissus species included in red lists. Values were obtained from standard floras of the regions. When these data are reported as ranges, the mean of the extreme values is considered

\begin{tabular}{|c|c|c|c|}
\hline & \multirow[b]{2}{*}{$\mathrm{n}$} & \multicolumn{2}{|c|}{ Longitud de la flor } \\
\hline & & Absoluta (mm) & $\begin{array}{l}\text { Relativa } \\
\text { (longitud flor / longitud planta) }\end{array}$ \\
\hline \multicolumn{4}{|l|}{ Lista roja de Andalucía ${ }^{1}$} \\
\hline En peligro de extinción & 4 & $34,0 \pm 7,5$ & $0,098 \pm 0,063$ \\
\hline Vulnerable & 2 & $18,7 \pm 4,0$ & $0,074 \pm 0,016$ \\
\hline Total amenazadas & 6 & $28,9 \pm 10,0$ & $0,090 \pm 0,051$ \\
\hline No amenazadas & 13 & $22,3 \pm 4,7$ & $0,150 \pm 0,095$ \\
\hline \multicolumn{4}{|l|}{ Lista roja de Marruecos ${ }^{2}$} \\
\hline Muy raras & 6 & $34,1 \pm 14,4$ & $0,174 \pm 0,069$ \\
\hline Raras & 6 & $28,9 \pm 10,3$ & $0,127 \pm 0,066$ \\
\hline Total raras & 12 & $31,5 \pm 12,3$ & $0,150 \pm 0,069$ \\
\hline No raras & 10 & $22,1 \pm 7,8$ & $0,118 \pm 0,101$ \\
\hline
\end{tabular}

${ }^{1}$ (Blanca et al. 1999a, 1999b); ${ }^{2}$ (Fennane \& Ibn-Tattou 1998) 
por tanto sabemos que es monomórfica, autocompatible pero con un amplio espectro de insectos polinizadores, que son necesarios para que haya producción de semillas. Sin embargo, no aparecen datos de la morfología floral que permitan saber si la especie presenta hercogamia de aproximación; no obstante ésta parece ser la situación, homogénea en todas las especies examinadas de esa sección (Barrett et al. 1996, Fig. 1). De esta forma tenemos cierta garantía de que esta especie conforma con las características propuestas como ancestrales en la ruta evolutiva del modelo de Lloyd \& Webb (1992a). De las restantes especies de esa lista roja prácticamente no se puede obtener información relevante. En algún caso hubiera sido especialmente interesante, por ejemplo en $N$. tortifolius, híbrido alopoliploide, estable y muy aislado, entre especies de la sección Tazettae, en la que predomina el dimorfismo estilar. Finalmente, en las dos especies consideradas relativamente menos amenazadas en la lista, N. fernandesii y $N$. viridiflorus, la información es confusa. Del primero se menciona que es heterostilo (¿distilo?), lo que sería un descubrimiento interesante que merecería documentarse cuantitativamente. Más probablemente esta aseveración sea una transcripción de la obra de Fernandes (1964) que ha sido puesta en duda por su nula base morfométrica, con lo que debe referirse a dimorfismo estilar (Barrett et al. 1996). De $N$. viridiflorus no se aclara su morfología floral, aunque ésta ya había sido medida exhaustivamente y confirmado su monomorfismo (Barrett et al. 1996, p. 348). Sobre el sistema de reproducción de estas dos especies se aporta poca información o no está contrastada (¿incompatibilidad esporofítica en N. fernandesii?), aunque ya se sabía que $N$. viridiflorus es autoincompatible (Barrett et al. 1996), pero no se conoce de qué tipo se trata ${ }^{1}$. Igualmente, todavía no hay disponible información fiel sobre los polinizadores de estas especies.

Evidentemente las críticas comentadas más arriba sobre los criterios de inclusión de especies y sobre la escasez o imprecisión de la información contenida para éstas no desvirtúan la utilidad de las listas rojas mencionadas, desde el momento en que su objetivo es de carácter más general y preliminar. Sin embargo, es conveniente que se vayan añadiendo requerimientos que permitan su refinamiento y que se contraste la información biológica ofrecida. Desde este punto de vista la

${ }^{1}$ Existe una versión posterior, no reconocida como tal, de esta obra en la que se han corregido algunos datos de estas especies. utilización de Narcissus como modelo para examinar la utilidad de las listas para conservar la diversidad dentro de un grupo de plantas se ha mostrado apropiada.

Finalmente, cuando se publican listas rojas de especies es casi inevitable que surjan discrepancias y/o propuestas alternativas referidas a especies concretas y basadas en la experiencia personal de diferentes investigadores. Aunque la subjetividad de tales planteamientos debe solventarse exclusivamente con datos contrastables, deben mencionarse algunos casos que apuntan a una consideración de mayor necesidad de conservación:

Narcissus humilis $(=N$. cavanillesii) es relativamente frecuente pero en un área muy reducida y en hábitats muy vulnerables (zonas bajas sometidas a cultivo). Su posición evolutiva es incierta. En lo que se refiere a evolución de la heterostilia representa un estadio basal, pero esta situación puede ser derivada. Su morfología floral es la más diferenciada del género. Si bien su protección en todo el territorio es difícil, al menos su presencia en ciertas áreas marginales debe apoyar la conservación de éstas.

Narcissus gaditanus es probablemente la especie que más ha reducido su distribución en el sur de la Península Ibérica, como consecuencia de su hábitat, arenas litorales, que es uno de los más amenazados por la expansión de las actividades turísticas. Además, presenta una morfología floral muy particular, con un tubo floral muy curvado, cuyo significado biológico se desconoce.

Narcissus cuatrecasasii no es excesivamente raro y aunque no está incluido en las listas rojas, su hábitat típico (zonas de bosque esclerófilo y de coníferas en general por encima de $1.000 \mathrm{~m} \mathrm{de}$ altitud) suele coincidir con parques naturales en Andalucía, donde se concentra su distribución. Aunque de forma indirecta ello aumenta sus posibilidades de conservación, debería considerarse su situación específicamente por su papel aparentemente importante en la evolución del polimorfismo floral en el género, al representar potencialmente la situación intermedia hacia la distilia. Especialmente importante es la única población encontrada recientemente en el Rif occidental (Benabid 2000), muy cerca de la única conocida de $N$. albimarginatus, a su vez la única especie distila conocida en el género.

Narcissus albimarginatus; aunque está recogido en la lista roja marroquí, lo es sólo por su rareza geográfica (endemismo muy restringido) y sería muy conveniente añadir la información evolutiva que proporciona, al representar un paso clave en el modelo de evolución de la distilia más arriba detallado. En la actualidad esta muy ame- 
nazado por el sobrepastoreo (ha sido muy díficil encontrar flores o frutos en los tres últimos años, aunque el número de plantas es elevado) en la única localidad conocida. Nuestra percepción de las fuerzas selectivas que modelan la evolución del polimorfismo floral en este grupo de plantas ha dependido mucho de haber podido muestrear algunas flores de esta especie, pero todavía queda mucho por conocer (polinizadores, sistema de cruzamiento).

Es de esperar que en una futura lista roja de la flora de Portugal sean tenidos en cuenta $N$. calcicola y $N$. scaberulus, ambos endémicos de ese país y además pertenecientes a la sección Apodanthae, la de más significado para el esclarecimiento de la evolución del polimorfismo, aunque el papel particular de estas especies está por determinar.

Todas las consideraciones anteriores tienen la apariencia de afectar sólo a grupos de plantas muy reducidos y a procesos muy particulares. Sin embargo, incluso desde el punto de vista del proceso de la evolución de la heterostilia pueden tener implicaciones más amplias porque todavía hay muchos grupos de plantas en los que el polimorfismo floral no está estudiado y es de gran potencial explicativo, por ejemplo en Lithodora (Boraginaceae), donde aparecen especies morfológicamente distilas y otras dimórficas, pero con posiciones de anteras diferentes en los morfos (Luis Navarro, comunicación personal). Sin embargo, el objetivo último de la evaluación realizada sobre Narcissus ha sido ejemplificar la necesidad de incorporar toda la información potencialmente disponible en las especies para estimar mejor su contribución a la diversidad biológica y su conservación.

\section{AGRADECIMIENTOS}

La mayor parte de los resultados propios mostrados forman parte de los Proyectos PB95-551 y PB98-1144 de la DGESIC. Un gran número de colegas ha ayudado a localizar o conseguir muestras de poblaciones de diferentes especies. Angela Baker amablemente cedió datos inéditos. Las discusiones con Spencer Barrett han sido una fuente constante de inspiración, mientras que Arndt Hampe y Fernando Ojeda aportaron sus ideas al diseño de algunos de los experimentos comentados. Rodrigo Tavera realizó los dibujos de las flores en la Fig. 1. Los comentarios de Begoña García, Arndt Hampe y Pablo Vargas sobre versiones previas del manuscrito han sido de mucha utilidad. Finalmente, debo agradecer a Mary Kalin-Arroyo y a Anna Traveset su invitación para participar en el Taller CYTED "Aspectos teóricos de la biología reproductiva de plantas y su relevancia para la conservación" y a todos los colegas participantes por el ambiente creado en este taller.

\section{LITERATURA CITADA}

ARROYO J \& SCH BARRETT (2000) Discovery of distyly in Narcissus (Amaryllidaceae). American Journal of Botany 87: 748-751.

ARROYO J \& A DAFNI (1995) Variations in habitat, season, flower traits and pollinators in dimorphic Narcissus tazetta L. (Amaryllidaceae) in Israel. New Phytologist 129: 135-146.

ARROYO J \& T MARAÑÓN (1990) Community ecology and distributional spectra of Mediterranean shrublands and heathlands in southern Spain. Journal of Biogeography 17: 163-176.

BAKER AM, JD THOMPSON \& SCH BARRETT (2000a) Evolution and maintenance of stigma-height dimorphism in Narcissus. I. Floral variation and stylemorph ratios. Heredity 84: 502-513.

BAKER AM, JD THOMPSON \& SCH B ARRETT (2000b) Evolution and maintenance of stigma-height dimorphism in Narcissus. II. Fitness comparisons between style morphs. Heredity 84: 514-524.

BARRETT SCH (ed) (1992) Evolution and function of heterostyly. Springer-Verlag, Berlin, Germany. 279 pp.

BARRETT SCH, WW COLE, J ARROYO, MB CRUZAN \& DG LLOYD (1997) Sexual polymorphisms in Narcissus triandrus (Amaryllidaceae): is this species tristylous? Heredity 78: 135-145.

BARRETT SCH, DG LLOYD \& J ARROYO (1996) Stylar polymorphisms and the evolution of heterostyly in Narcissus (Amaryllidaceae). En: Lloyd DG \& SCH Barrett (eds) Floral biology, studies on floral evolution in animal-pollinated plants: 339-376. Chapman \& Hall, New York, New York.

BARRETT SCH, LK JESSON \& AM BAKER (2000) The evolution and function of stylar polymorphisms in flowering plants. Annals of Botany 85 (Supplement A): 253-265.

BATEMAN AJ (1952) Trimorphism and selfincompatibility in Narcissus. Nature 170: 496-497.

BENABID A (2000) Contribuciones a la flora vascular de Marruecos. 1. Nouveaux materiaux pour la flore du Maroc (fasc. 5) 199: Narcissus cuatrecasii. Acta Botanica Malacitana 25: 255.

BININDA-EMONDS ORP, DP VÁZQUEZ \& LL MANNE (2000) The calculus of biodiversity: integrating phylogeny and conservation. Trends in Ecology and Evolution 15: 92-94.

BLANCA G, B CABEZUDO, JE HERNÁNDEZ-BERMEJO, CM HERRERA, J MOLERO MESA, J MUÑOZ \& B VALDÉS (1999a) Libro rojo de la flora silvestre amenazada de Andalucía. Tomo I. Especies en peligro de extinción. Consejería de Medio Ambiente, Junta de Andalucía, Sevilla, España. 302 pp. 
BLANCA G, B CABEZUDO, JE HERNÁNDEZ-BERMEJO, CM HERRERA, J MUÑOZ \& B VALDÉS (1999b) Libro rojo de la flora silvestre amenazada de Andalucía. Tomo II. Especies vulnerables. Consejería de Medio Ambiente, Junta de Andalucía, Sevilla, España. $375 \mathrm{pp}$.

BLANCHARD JW (1990) Narcissus, a guide to wild daffodils. Alpine Garden Society, Surrey, United Kingdom. 201 pp.

BRICKELL C (ed) (1994) Gardeners' Encyclopedia of plants and flowers. The Royal Horticultural Society, Dorling Kindersley, London, England. 640 pp.

CASTRO I, JC MORENO, CJ HUMPHRIES \& PH WILLIAMS (1996) Strengthening the Natural and National Park system of Iberia to conserve vascular plants. Botanical Journal of the Linnean Society 121 : 189-206.

CHARLESWORTH D \& B CHARLESWORTH (1979) A model for the evolution of distyly. American Naturalist 114: 467-498.

DARWIN C (1877) The different forms of flowers on plants of the same species. John Murray, London, England. $352 \mathrm{pp}$.

DIOSDADO-FERNÁNDEZ JC \& JE PASTOR-DÍAZ (1997) Contribución al atlas cromosómico de la flora vascular de Andalucía oriental. Universidad de Sevi1la, Sevilla, España. 153 pp.

DOBSON HEM, J ARROYO, G BERGSTRÖM \& I GROTH (1997) Interspecific variation in floral fragrances within the genus Narcissus (Amaryllidaceae). Biochemical Systematics and Ecology 25: 685-706.

DULBERGER R (1964) Flower dimorphism and selfincompatibility in Narcissus tazetta L. Evolution 18: 361-363.

DULBERGER R (1992) Floral polymorphisms and their functional significance in the heterostylous syndrome. En: Barrett $\mathrm{SCH}$ (ed) Evolution and function of heterostyly: 41-84. Springer-Verlag, Berlin, Germany.

FENNANE M \& M IBN-TATTOU (1998) Catalogue des plantes vasculaires rares, menacées ou endémiques du Maroc. Bocconea 8: 5-243.

FERNANDES A (1935) Remarque sur l'hétérostylie de Narcissus triandrus et $N$. reflexus. Boletim da Sociedade Broteriana Série 2, 10: 5-15.

FERNANDES A (1964) Contribution à la connaissance de le génétique de l'hétérostylie chez le genre Narcissus L. Résusltats de quelques croisements. Boletim da Sociedade Broteriana, Série 2, 38: 81-96.

FERNANDES A (1975) L'evolution chez le genre Narcissus L. Anales del Instituto Botánico Cavanilles 32: 843-872.

FIEDLER PL \& JJ AHOUSE (1992) Hierarchies of cause: Towards an understanding of rarity in vascular plant species. En: Fiedler PL \& SK Jain (eds) Conservation biology: 23-47. Chapman \& Hall, New York, New York.

GANDERS FR (1979) The biology of heterostyly. New Zealand Journal of Botany 17: 607-635.

GEBER MA, TE DAWSON \& LF DELPH (eds) (1999) Gender and sexual dimorphism in flowering plants. Springer, Berlin, Germany. 305 pp.
GILBERT LE (1980) Food web organization and the conservation of Neotropical diversity. En: Soulé ME \& BA Wilcox (eds) Conservation biology: an evolutionary-ecological perspective. Sinauer Associates, Sunderland, Massachusetts.

GIVEN DR (1994) Principles and practice of plant conservation. Chapman \& Hall, London, United Kingdom. 292 pp.

GOLDBLATT P (1993) The woody Iridaceae: Nivenia, Klattia, and Witsenia. Timber Press, Portland, Oregon. $128 \mathrm{pp}$.

GOLDBLATT P \& P BERNHARDT (1990) Pollination biology of Nivenia (Iridaceae) and the presence of heterostylous self-compatibility. Israel Journal of Botany 39: 93-111.

GÓMEZ-CAMPO C (1987) Libro rojo de especies vegetales amenazadas de España peninsular e Islas Baleares. Instituto para la Conservación de la Naturaleza, Madrid, España. 676 pp.

HARTL DL (1988) A primer of population genetics. Segunda edición. Sinauer Associates, Sunderland, Massachusetts. 305 pp.

HERRERA CM (1989) Sobre el vicio del tamaño y la práctica de la conservación. Quercus 35: 43-48.

HERRERA CM (1995) Floral biology, microclimate, and pollination by ectothermic bees in an early blooming herb. Ecology 76: 218-228.

HERRERA CM (1996) Floral traits and plant adaptation to Insect pollinators: a devil's advocate approach. En: Lloyd DG \& SCH Barrett (eds) Floral biology: studies on floral evolution in animal-pollinated plants: 6587. Chapman \& Hall, New York, New York.

LLOYD DG \& CJ WEBB (1992a) The evolution of heterostyly. En: Barrett SCH (ed) Evolution and function of heterostyly: 151-178. Springer-Verlag, Berlin, Germany.

LLOYD DG \& CJ WEBB (1992b) The selection of heterostyly. En: Barrett SCH (ed) Evolution and function of heterostyly: 179-207. Springer-Verlag, Berlin, Germany.

LLOYD DG, CJ WEBB \& R DULBERGER (1990) Heterostyly in species of Narcissus (Amaryllidaceae), Hugonia (Linaceae) and other disputed cases. Plant Systematics and Evolution 172: 215-227.

MAIRE R (1959) Flore de l'Afrique du Nord, volume VI. Paul Lechevalier, Paris, France. 397 pp.

MATTHIES D \& P POSCHOLD (2000) The biological flora of central Europe. Aims and Concepts. Flora 195: 116-122.

MAY RM (1990) Taxonomy as destiny. Nature 347: 129130.

MORENO-SAIZ JC \& H SAINZ-OLLERO (1992) Atlas corológico de las monocotiledóneas endémicas de la Península Ibérica y Baleares. Instituto para la Conservación de la Naturaleza, Madrid, España. 354 pp.

OJEDA F, J ARROYO \& T MARAÑÓN (1995) Biodiversity components and conservation of Mediterranean heathlands in southern Spain. Biological Conservation 72: 61-72.

ORNDUFF R (1988) Distyly and monomorphism in Villarsia (Menyanthaceae): some evolutionary considerations. Annals of the Missouri Botanical Garden 75: 761-767. 
ORNDUFF R (1992) Historical perspectives on heterostyly. En: Barrett SCH (ed) Evolution and function of heterostyly: 32-39. Springer-Verlag, Berlin, Germany.

PAINE RT (1966) Food web complexity and species diversity. American Naturalist 100: 65-75.

PRESSEY RL, CJ HUMPHRIES, CR MARGULES, RI VANE-WRIGHT \& PH WILLIAMS (1993) Beyond opportunism: key principles for systematic reserve selection. Trends in Ecology and Evolution 8: 124128.

PRIMACK R (1993) Essentials of conservation biology. Sinauer Associates, Sunderland, Massachusetts. 564 pp.

RHS (1998) International Daffodil Register and Classified List (1998) http://www.rhs.org.uk/science/ RegisterPages/daff7.asp

RICHARDS AJ (1997) Plants breeding systems. Segunda edición. Chapman \& Hall, London, United Kingdom. $529 \mathrm{pp}$.

RICHARDS AJ (1998) Lethal linkage and its role in the evolution of plant breeding systems. En: Owens SJ \& PJ Rudall (eds) Reproductive biology: 71-83. Royal Botanic Gardens, Kew, United Kingdom.

SAGE TL, F STRUMAS, WW COLE \& SCH BARRETT (1999) Differential ovule development following selfand cross- pollination: the basis of self-sterility in Narcissus triandrus (Amaryllidaceae). American Journal of Botany 86: 855-870.

SCHOU O \& M PHILIPP (1984) An unusual heteromorphic incompatibility system. III. On the genetic control of distyly and self-incompatibility in Anchusa officinalis L. (Boraginaceae). Theoretical and Applied Genetics 68: 139-144.

SOLBRIG OT (1991) Biodiversity: scientific issues and collaborative research proposals. MAB Digest 9, UNESCO, Paris, France. 77 pp.
STEBBINS GL (1970) Adaptive radiation of reproductive characteristics in angiosperms. I. Pollination mechanisms. Annual Review of Ecology and Systematics 1: 307-326.

VALDÉS B (1981) Notas sobre boragináceas españolas. I. Lithodora prostrata (Loisel.) Griseb. y L. diffusa (Lag.) I.M. Johnston. Boletim da Sociedade Broteriana série 2, 53: 1331-1340.

VALDÉS B (1987) Narcissus. En: Valdés B, S Talavera \& EF Galiano (eds) Flora vascular de Andalucía occidental, volumen 3: 463-474. Ketres, Barcelona, España.

VANE-WRIGHT RI, CJ HUMPHRIES \& PH WILLIAMS (1991) What to protect? Systematics and the agony of choice. Biological Conservation 55: 235-254.

VVAA (2000) Lista roja de flora vascular española (valoración según categorías UICN). Conservación Vegetal 6 (extra): 11-38.

WEBB DA (1980) Narcissus. En: Tutin TG, VH Heywood, NA Burges, DM Moore, DH Valentine, SM Walters \& DA Webb (eds) Flora europea, volumen 5: 78-84. Cambridge University Press, Cambridge, United Kingdom.

WESTOBY M, M LEISHMAN \& J LORD (1996) Comparative ecology of seed size and dispersal. Philosophical Transactions of the Royal Society of London B 351: 1309-1318.

WILLIAMS PH \& CH HUMPHRIES (1993) Biodiversity, taxonomic relatedness and endemism in conservation. En: Forey PL, CJ Humphries \& RI Vane-Wright (eds) Systematic and conservation evaluation: 1-16. The Systematics Association, Oxford University Press, Oxford, United Kingdom. 